\title{
Nematodos de los arrecifes de Isla Mujeres y Banco Chinchorro, Quintana Roo, México
}

\author{
Nematodes from Isla Mujeres and Banco Chinchorro reefs, Quintana Roo, Mexico \\ Alberto de Jesús-Navarrete ${ }^{1}$ \\ ${ }^{1}$ Departamento de Aprovechamiento y Manejo de Recursos Acuático \\ El Colegio de la Frontera Sur-Unidad Chetumal, A. P. 424 Chetumal, Quintana Roo, México, C. P. 77900 \\ alberto@ecosur-qroo.mx
}

\begin{abstract}
In order to know the composition of free living marine nematodes, samples were collected in three sites at Isla Mujeres and four sites at Banco Chinchorro, Quintana Roo, Mexico. At Isla Mujeres, 34 genera were identified corresponding to 17 families and three orders. Chromadorida was the order best represented with 20 genera, followed by the order Monhysterida with eight genera and the order Enoplida with six genera. Family Desmodoridae had the highest number of genera (five). The most abundant genera (individuals $0.001 \mathrm{~m}^{-2}$ ) were: Terchellingia longicaudata (34), Spirinia parasitifera (23), Croconema cincta (22), Eubostrichus porosum (20), and Xyala riemmani (18). Station E2 had the high nematode abundance (125 individuals $0.001 \mathrm{~m}^{-2}$ ), whereas station E3 had the lowest amount (85 individuals $0.001 \mathrm{~m}^{-2}$ ). At Banco Chinchorro, nematofauna corresponded to three orders, 17 families, and 49 genera. Chromadorida had the highest number of families and genera (9 and 34 respectively). Enoplida had five families and eight genera, whereas Monhysterida had three families and seven genera. Desmodoridae showed the highest number of genera (11). Croconema cincta, presented the highest abundance (36 individuals $0.001 \mathrm{~m}^{-2}$ ) followed in descendent order by Dorylaimopsis sp. 1 (24), Croconema otti (22), Paracomesoma longispiculum and Trypilodes marinus with 20, respectively. $\mathrm{CN}$ had the highest abundance by station (243 individuals $0.001 \mathrm{~m}^{-2}$ ), whereas $\mathrm{CL}$ had the lowest (37 individuals $0.001 \mathrm{~m}^{-2}$ ). In both localities, dominant nematodes had small and annulated bodies with many somatic setae, typical of sandy environments. All species are new records for Mexico.
\end{abstract}

Key words: Caribbean reefs, free living nematodes, meiofauna.
Resumen.- Para conocer la composición de los Nematoda de vida libre, se recolectaron muestras en tres sitios en Isla Mujeres y cuatro sitios en Banco Chinchorro, Quintana Roo, México. En Isla Mujeres, se identificaron 34 géneros que correspondieron a 17 familias y tres órdenes. El orden Chromadorida, fue el mejor representado con 20 géneros, seguido por el orden Monhysterida con ocho géneros y el orden Enoplida con seis. La familia Desmodoridae tuvo el mayor número de géneros (cinco). Los géneros más abundantes (individuos $0,001 \mathrm{~m}^{-2}$ ) fueron: Terchellingia longicaudata (34), Spirinia parasitifera (23), Croconema cincta (22), Eubostrichus porosum (20) y Xyala riemmani (18). La estación E2 tuvo la mayor abundancia de nematodos (125 individuos $0,001 \mathrm{~m}^{-2}$ ), en tanto que la $\mathrm{E} 3$ tuvo la menor cantidad ( 85 individuos $0,001 \mathrm{~m}^{-2}$ ). En Banco Chinchorro, la nematofauna quedó representada por tres órdenes, 17 familias y 49 géneros. El orden Chromadorida tuvo el mayor número de familias y géneros ( 9 y 34 respectivamente). Enoplida tuvo cinco familias y ocho géneros mientras el orden Monhysterida tuvo tres familias y siete géneros. La familia Desmodoridae mostró el más alto número de géneros (11). Croconema cincta presentó la mayor abundancia (36 individuos $0,001 \mathrm{~m}^{-2}$ ) y le siguieron, en orden decreciente, Dorylaimopsis sp. 1 (24), Croconema otti (22), Paracomesoma longispiculum y Trypilodes marinus con 20, respectivamente. $\mathrm{CN}$ presentó la mayor abundancia por estación (243 individuos $0,001 \mathrm{~m}^{-2}$ ), mientras que CL tuvo la menor abundancia $\left(37\right.$ individuos $0,001 \mathrm{~m}^{-2}$ ). En ambas localidades los nematodos dominantes tuvieron cuerpos pequeños, anillados y con muchas setas somáticas típicas de ambientes arenosos. Todas las especies halladas son nuevos registros para México.

Palabras clave: Arrecifes del caribe, nematodos de vida libre, meiofauna 


\section{Introducción}

Las poblaciones de nematodos del gran Caribe son poco conocidas. La composición de Nematoda en aguas profundas en Venezuela y Puerto Rico fue estudiada por Tietjen $(1984,1989)$. Aspectos de taxonomía y composición de la nematofauna de Cuba fueron tratados por Botosaneau (1970) y Andrassy (1973). Wagenaar Hummerlinck (1977) investigó la meiofauna de Martinica y de las Islas Guadeloupe, RenaudMornant \& Gourbault (1981), y Boucher \& Gourbault (1990) estudiaron la distribución de la meiofauna y su relación con las características ambientales. Decramer \& Gourbault (1986, 1987), Gourbault \& Decramer (1986, 1987, 1988) y Gourbault \& Vincx (1990) describieron las nuevas especies de Guadeloupe.

En México existen pocos trabajos sobre ecología y aún menos sobre taxonomía de nematodos. Castillo \& Lambshead (1992) revisaron el género Elzalia y describieron tres nuevas especies recolectadas en el Golfo de México. En 1993, Castillo \& Decramer describieron a Cheironchus paravorax y redescribieron a Cheironchus vorax encontrados en la sonda de Campeche. En esta misma área, se identificaron 117 especies relacionando su distribución con la concentración de hidrocarburos totales en los sedimentos (de Jesús-Navarrete 1993a). En Quintana Roo, la composición de los nematodos de la laguna de Buenavista fue realizada por de Jesús-Navarrete (1993b). En la bahía de Chetumal, se describió la nematofauna asociada a la zona urbana de la ciudad (de Jesús-Navarrete \& Herrera-Gómez 1999, 2002). Sin embargo, no existe información sobre la nematofauna de los arrecifes del caribe mexicano. Este trabajo tiene como objetivo contribuir al conocimiento de la nematofauna del caribe mexicano y brindar información sobre las especies presentes en el área.

\section{Materiales y métodos}

\section{Área de estudio}

En julio de 1995 se recolectaron muestras de sedimentos en tres de los vértices del polígono del Parque Marino Nacional Costa Occidental de Isla Mujeres: E1 $\left(21^{\circ} 16^{\prime} \mathrm{N}, 86^{\circ} 46^{\prime} 30^{\prime \prime} \mathrm{W}\right)$, E2 $\left(21^{\circ} 16^{\prime} \mathrm{N}\right.$, $\left.86^{\circ} 45^{\prime} \mathrm{W}\right)$ y E3 $\left(21^{\circ} 13^{\prime} \mathrm{N}, 86^{\circ} 45^{\prime} \mathrm{W}\right)$. La profundidad varió de seis a diez metros. En junio de 1996 se recolectaron muestras en cuatro sitios de la Reserva de la Biosfera Banco Chinchorro Cayo Lobos (CL) $\left(18^{\circ} 23^{\prime} \mathrm{N}, 8^{\circ} 22^{\prime} \mathrm{W}\right)$, Cayo Centro (CC) $\left(18^{\circ} 23^{\prime} \mathrm{N}\right.$, $\left.87^{\circ} 19^{\prime} \mathrm{W}\right)$, Isla Che, (ICh) $\left(18^{\circ} 29^{\prime} \mathrm{N}, 87^{\circ} 26^{\prime} \mathrm{W}\right)$ y Cayo Norte (CN), $\left(18^{\circ} 43^{\prime} \mathrm{N}, \quad 87^{\circ} 21^{\prime} \mathrm{W}\right)$ (Fig. 1). La profundidad varió de dos a veinte metros.

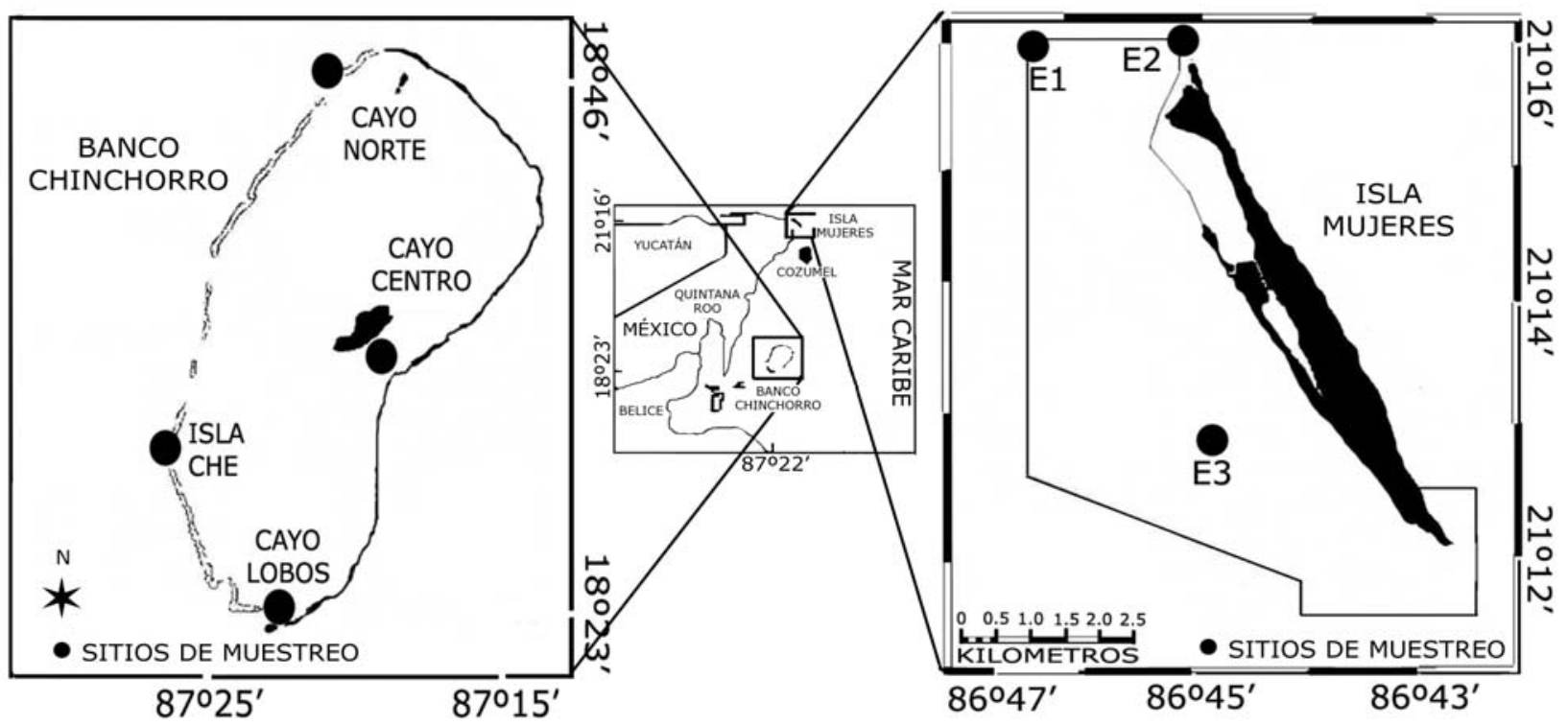

Figura 1

\section{Localización del área estudio}

Location of the study area 
Las muestras fueron recolectadas por duplicado, con un tubo de PVC de $5 \mathrm{~cm}$ de diámetro, que se enterró en el sedimento hasta una profundidad de 10 $\mathrm{cm}$. La fauna se fijó con una solución de formol al 10\% caliente $\left(60^{\circ} \mathrm{C}\right)$. Una muestra se utilizó para el análisis granulométrico (Buchanan 1984) y materia orgánica (Dean 1974), mientras que la otra se usó para el análisis de los nematodos. Adicionalmente, en cada sitio se midieron temperatura, oxígeno disuelto y salinidad con un conductivímetro modelo YSI 86). La meiofauna se extrajo por elutriación y los nematodos se separaron manualmente del resto de la meiofauna, se montaron en laminillas de vidrio y se determinaron a nivel de género con las claves de Tarjan (1980) y Platt \& Warwick (1983). Para la identificación específica se usaron las descripciones originales. Los organismos identificados se depositaron en la Colección de Nematodos Acuáticos del Colegio de la Frontera Sur-Unidad Chetumal. La abundancia se expresó en individuos $0,001 \mathrm{~m}^{-2}$.

\section{Resultados}

Se procesó un total de 735 individuos para las dos localidades. En Isla Mujeres se identificaron 34 géneros, que corresponden a 17 familias y tres órdenes. El orden Chromadorida fue el mejor representado con 20 géneros; siguió en importancia el orden Monhysterida con ocho géneros y finalmente el orden Enoplida con seis géneros. Dentro de los chromadoridos, la familia Desmodoridae presentó el mayor número de géneros (cinco). Los cinco géneros más abundantes fueron: Terchellingia longicaudata (34 individuos $0,001 \mathrm{~m}^{-2}$ ), Spirinia parasitifera (23), Croconema cinctum (22), Eubostrichus porosum (20) y Xyala riemmani con 18 individuos $0,001 \mathrm{~m}^{-2}$. La estación E2 tuvo la mayor abundancia de nematodos (125), en tanto que la E3 tuvo la menor cantidad (85) (Tabla 1).

Tabla 1

Nematodos marinos de vida libre de los arrecifes: Parque Nacional Costa Occidental de Isla Mujeres y Banco Chinchorro (Individuos $\mathbf{0 , 0 0 1} \mathrm{m}^{-2}$ )

Free living marine nematodes from reefs: Parque Nacional Costa Occidental of Isla Mujeres and Banco Chinchorro (Individuals $0.001 \mathrm{~m}^{-2}$ )

\begin{tabular}{|c|c|c|c|c|c|c|c|}
\hline \multirow{2}{*}{$\begin{array}{l}\text { Localidad } \\
\text { Género/Estación }\end{array}$} & \multicolumn{3}{|c|}{ Isla Mujeres } & \multicolumn{4}{|c|}{ Banco Chinchorro } \\
\hline & E1 & E2 & E3 & $\mathbf{C L}$ & CC & Ich & $\mathbf{C N}$ \\
\hline \multicolumn{8}{|l|}{ Enoplida } \\
\hline \multicolumn{8}{|l|}{ Enchelidiidae } \\
\hline Polygastrophora sp. 1 & & 1 & & & & & 8 \\
\hline \multicolumn{8}{|l|}{ Enoplidae } \\
\hline Enoplus communis (Bastian, 1865) & & & & & & & 3 \\
\hline \multicolumn{8}{|l|}{ Oncholaiidae } \\
\hline Viscosia papillata (Chitwood, 1961) & & & & & & & 3 \\
\hline \multicolumn{8}{|l|}{ Oxystominidae } \\
\hline Halalaimus parafletcheri (Keppner, 1992) & 5 & 5 & & & 2 & & \\
\hline \multicolumn{8}{|l|}{ Thorascostomopsidae } \\
\hline Enoploides & & 1 & & 2 & & 5 & \\
\hline Mesacanthion sp.1 & & & & 2 & & 2 & \\
\hline Mesacanthoides sp. 1 & & & & & & 15 & \\
\hline Oxyonchus sp.1 & & 1 & & & & & \\
\hline \multicolumn{8}{|l|}{ Trypiloidiidae } \\
\hline Bathylaimus australis (Cobb, 1984) & 9 & & 3 & & & & \\
\hline Bathylaimus longicorpus (Keppner, 1988) & 7 & & & & & & 11 \\
\hline Trypiloides marinus (Butschli, 1874) & & & & 8 & 5 & & 7 \\
\hline \multicolumn{8}{|l|}{ Chromadorida } \\
\hline \multicolumn{8}{|l|}{ Ceramonematidae } \\
\hline Ceramonema sculpturatum (Chitwood, 1936) & & 7 & & & & & 4 \\
\hline Pselionema longissimum (Gerlach, 1963) & & & & & 6 & & \\
\hline
\end{tabular}


Continuación Tabla 1

\begin{tabular}{lcccccc}
\hline Localidad & \multicolumn{3}{c}{ Isla Mujeres } & \multicolumn{3}{c}{ Banco Chinchorro } \\
Género/Estación & E1 & E2 & E3 & CL & CC & Ich \\
\hline
\end{tabular}

Comesomatidae

Dorylaimopsis sp. 1

Paracomesoma longispiculum (Tim, 1961)

Paracomesoma sp. 1

14

Cyatholaimidae

Cyatholaimus sp 1.

Marylinnia sp. 1

Nannolaimus fusus (Gerlach, 1956)

Paracyatholaimus sp. 1

Pomponema sp. 1

4

Chromadoridae

Dichromadora geophila (de Man, 1876)

Euchromadora sp. 1

Metachromadoroides sp. 1

Neochromadora sp. 1

Parapinnanema sp. 1

Ptycholaimellus jacobi (Jensen \& Nehering, 1991)

Rhips sp. 1

Spilophorella paradoxa (de Man, 1888)

Desmodoridae

Bolbonema sp.1

Croconema cinctum (Cobb, 1920)

Croconema otti (Gourbault \& Vincx, 1990)

Desmodora brevicollis (Cobb, 1920)

Desmodora sp. 3

Desmodora sp. 4

Eubostrichus hopperi (Muthumbi et al.,1995).

Eubostrichus porosum (Hopper \& Cefalu, 1973)

Leptonemella sp. 1

Metachromadora sp. 1

Onyx sp. 1

Paradesmodora sp. 1

Spirinia parasitifera (Bastian, 1865)

Desmoscolecidae

Desmoscolex laevis (Kreiss, 1928)

Desmoscolex cosmopolites (Tim, 1970)

Tricoma hoperi (Tim, 1970)

Draconematidae

Dracognomus sp. 1

Epsilonematidae

Epsilonema sp. 1

Ethmolaimidae

Filitonchus sp. 1

Gomphionema sp. 1

$18 \quad 2$

4

5

11

12

5

13

5

10

Microlaimidae

Microlaimus sp.1

Monopostiidae

Monoposthia hexalata (Chitwood, 1936)

Monoposthia mirabilis (Schuktz, 1932)
9

1

3
1

2

2

3

4

4

7

2

9

2

5

5

4

3

2

2

6

1

5

$5 \quad 8$

$\begin{array}{lllll}1 & 4 & 7 & 1 & 5\end{array}$ 


\begin{tabular}{|c|c|c|c|c|c|c|c|}
\hline \multirow{2}{*}{$\begin{array}{l}\text { Localidad } \\
\text { Género/Estación }\end{array}$} & \multicolumn{3}{|c|}{ Isla Mujeres } & \multicolumn{4}{|c|}{ Banco Chinchorro } \\
\hline & E1 & E2 & E3 & CL & $\mathbf{C C}$ & Ich & $\mathbf{C N}$ \\
\hline \multicolumn{8}{|l|}{ Selachinematidae } \\
\hline Cheironchus vorax (Cobb, 1917) & & 2 & & & & & 4 \\
\hline Halichoanolaimus duodecimpapillatus (Tim, 1952) & & & & & & 5 & \\
\hline Latronema sp. 1 & & & & & & & 5 \\
\hline Richtersia comansi (Soetaert y Vincx, 1987) & 1 & & 13 & & & & 6 \\
\hline \multicolumn{8}{|l|}{ Synonchiella sp. 1} \\
\hline \multicolumn{8}{|l|}{ Tarvaiidae } \\
\hline Tarvaia sp. 1 & & 4 & & & & & \\
\hline Paratarvaia sp. 1 & & & & 2 & & & \\
\hline \multicolumn{8}{|l|}{ Monhysterida } \\
\hline \multicolumn{8}{|l|}{ Axonolaimidae } \\
\hline Odontophora carrolli (Keppner, 1988) & 6 & 2 & & & 4 & & 8 \\
\hline Odontophora sp. 1 & & 3 & & & & & \\
\hline Parodontophora sp. 1 & & & & & & & 4 \\
\hline \multicolumn{8}{|l|}{ Diplopeltidae } \\
\hline Araeolaimus sp. 1 & & & & & & 5 & \\
\hline Diplopeltula sp. 1 & & 5 & & & & & \\
\hline \multicolumn{8}{|l|}{ Linhomoeidae } \\
\hline Didelta sp. 1 & & 4 & & & & & \\
\hline Terschellingia longicaudata (de Man, 1907) & 17 & & 17 & & & & 7 \\
\hline \multicolumn{8}{|l|}{ Xyalidae } \\
\hline Rhinchonema hirsutum (Hoper, 1961) & & & & 5 & & & 2 \\
\hline Theristus sp. 1 & & 1 & & & & & 4 \\
\hline Theristus sp. 2 & & & & & & 1 & \\
\hline Xyala riemanni (Boucher \& Helleouet, 1977) & 13 & 5 & & & & & \\
\hline Xyala striata $(\mathrm{Cobb}, 1929)$ & 9 & 1 & & & & & \\
\hline Número de organismos & 101 & 125 & 85 & 37 & 69 & 75 & 243 \\
\hline
\end{tabular}

E1= Estación 1, E2= Estación 2, E3= Estación 3, CN= Cayo Lobos, CL=Cayo Centro, Ich= Isla Che

En Banco Chinchorro, los nematodos quedaron representados en 17 familias, 3 órdenes y 49 géneros. El orden Chromadorida presentó la mayor cantidad de familias y de géneros (9 y 34 respectivamente). Siguieron en importancia el orden Enoplida con cinco familias y ocho géneros y el orden Monhysterida con tres familias y siete géneros. La familia Desmodoridae fue la que tuvo el mayor número de géneros (12). Croconema cinctum presentó la mayor abundancia (36 individuos $\left.0,001 \mathrm{~m}^{-2}\right), \mathrm{y}$ le siguieron en orden decreciente, Dorylaimopsis sp. 1 (24), Croconema otti (22), Paracomesoma longispiculum y Trypilodes marinus (20). CN presentó la mayor abundancia por estación (243 individuos $0,001 \mathrm{~m}^{-2}$ ), mientras que $\mathrm{CL}$ (37) tuvo la menor abundancia (37) (Tabla 1).

Los parámetros ambientales medidos indican aguas cálidas y típicas de los ambientes marinos tropicales. En Isla Mujeres, la temperatura mínima se registró en la estación E3 $\left(27,9{ }^{\circ} \mathrm{C}\right)$ mientras que la máxima correspondió a la estación E1 $\left(28,6^{\circ} \mathrm{C}\right)$. La salinidad estuvo por encima de las 35 ups en los tres sitios de muestreo. Las aguas fueron oxigenadas con valores de oxígeno disuelto superior a $4,2 \mathrm{mg} \mathrm{L}^{-1}$ en los tres sitios. Los sedimentos correspondieron a arenas finas con un tamaño medio de grano de 2,75 $\Phi$ en la escala de Wenworth y el contenido de materia orgánica fue menor al 3\% (Tabla 2).

En Banco Chinchorro se observaron temperaturas que variaron entre 28,5 y $28,8{ }^{\circ} \mathrm{C}$, mientras que la salinidad varió entre 36,1 y 36,4 ups. Al igual que en Isla Mujeres el oxígeno disuelto fue mayor a $4,2 \mathrm{mg} \mathrm{L}^{-1}$ en las cuatro estaciones de muestreo. Los sedimentos correspondieron a arenas medianas ( $\mathrm{CC}$ y $\mathrm{CN}$ ) y gruesas (CL e Ich) con tamaños medios de grano de $0,24 \Phi$ hasta 1,4 $\Phi$ en la escala de Wenworth. Los porcentajes de materia orgánica variaron de 13,1\% como mínimo en Ich, a $21,7 \%$ como máximo en $\mathrm{CN}$ (Tabla 2). 
Tabla 2

\section{Parámetros ambientales en el Parque Nacional Costa Occidental de Isla Mujeres y Banco Chinchorro}

Environmental parameters at Parque Nacional Costa Occidental of Isla Mujeres and Banco Chinchorro

ISLA MUJERES

\begin{tabular}{|c|c|c|c|c|}
\hline Estación/parámetro & E1 & E2 & E3 & \\
\hline Temperatura $\left({ }^{\circ} \mathrm{C}\right)$ & 28,6 & 28,4 & 27,9 & \\
\hline Salinidad (ups) & 35,8 & 35,9 & 36,0 & \\
\hline Oxígeno $\left(\mathrm{mg} \mathrm{L}^{-1}\right)$ & 4,3 & 4,29 & 4,2 & \\
\hline Tamaño medio de grano $(\Phi)$ & 2,75 & 2,3 & 2,75 & \\
\hline Tipo de sedimento & Arena fina & Arena fina & Arena fina & \\
\hline Materia orgánica (\%) & 1,76 & 1,96 & 2,14 & \\
\hline & \multicolumn{4}{|c|}{ BANCO CHINCHORRO } \\
\hline Estación/parámetro & CL & CC & Ich & $\mathbf{C N}$ \\
\hline Temperatura $\left({ }^{\circ} \mathrm{C}\right)$ & 28,6 & 28,8 & 28,5 & 28,7 \\
\hline Salinidad (ups) & 36,1 & 36,0 & 36,1 & 36,4 \\
\hline Oxígeno $\left(\mathrm{mg} \mathrm{L}^{-1}\right)$ & 4,2 & 4,3 & 4,5 & 4,34 \\
\hline Tamaño medio de grano $(\Phi)$ & 0,24 & 1,4 & 0,89 & 1,29 \\
\hline Tipo de sedimento & Arena gruesa & $\begin{array}{c}\text { Arena } \\
\text { mediana }\end{array}$ & Arena gruesa & $\begin{array}{c}\text { Arena } \\
\text { mediana }\end{array}$ \\
\hline Materia orgánica (\%) & 14,7 & 21,7 & 13,1 & 21,7 \\
\hline
\end{tabular}

E1= Estación 1, E2= Estación 2, E3= Estación 3, CL= Cayo Lobos, CC= Cayo Centro, Ich= Isla Che, CN= Cayo Norte

El $34,6 \%$ de los organismos recolectados corresponden a especies descritas en otras áreas del mundo. Considerando ambas localidades, el $100 \%$ de las especies son nuevos registros para México. En ambos sitios, dominaron los nematodos desmodoridos y presentaron tamaños pequeños, formas anilladas y abundantes setas somáticas, características que les permiten mantenerse "anclados" en sedimentos de arenas calcáreas medianas y gruesas poco consolidadas.

\section{Discusión}

Muchas de las especies encontradas en ambas localidades ya han sido reportadas en áreas con características similares. En la costa este de Florida, se ha reportado la presencia de Rhinchonema hirsuta (Hopper 1961), Xyala striata (Hopper 1963), Paracomesoma longispiculum (Hoper 1967), Bathylaimus australis, Viscosia papillata, Halichaonolaimus duodecimpapillatus, Spirinia parasitifera, Monophostia mirabilis, Spilophorella paradoxa, Terschellingia longicaudata (Wieser \& Hopper 1967). En las aguas de Florida, en el Golfo de México, se informó de la presencia de Halalaimus parafletcheri, Bathylaimus longicorpus, Odontophora carrolli (Keppner 1988, 1992), así como Ptycholaimellus jacobi (Jensen 1986). Aunque las condiciones ambientales entre el Golfo de México y el Mar Caribe son diferentes, aparentemente estas especies tienen un amplio rango de distribución. Todas estas especies extienden su ámbito de distribución hasta el caribe mexicano.

En la isla Guadeloupe, Boucher \& Gourbault (1990) informaron de la presencia de Spirinnia parasitifera, Terschellingia longicaudata y Nannolaimus fusus, que coinciden con especimenes reportados en este trabajo. Croconema otti fue descrita por Gourbault \& Vincx (1990) y fue encontrada en Banco Chinchorro. Así, Croconema otti y Nannolaimus fusus extienden su distribución hacia el oeste del caribe, en Banco Chinchorro. 
El resto de las especies se ha encontrado en áreas meridionales de América y Europa, como: Enoplus communis, Ceramonema sculpturatum, Eubostrichus hopperi, Monophosthia hexalata, que fueron encontradas en Carolina del Norte, (Chitwood 1934), en tanto que Tricoma hopperi fue hallada en Bermuda (Tim 1970).

En el Mediterráneo se informó de la presencia de Pselionema longisimum, (Gerlach 1953), Desmoscolex laevis, Desmoscolex cosmopolites (Tim 1970), Richtersia commansi (Soetaert \& Vincx 1987), mientras que en el Mar del Norte se recolectó Xyala riemanni (Boucher \& Helleouet 1977); estas especies estuvieron presente tanto en Isla Mujeres como en Banco Chinchorro. Croconema cinctum (Cobb 1920) fue encontrada en Jamaica y reportada en Brasil por Gerlach (1963), y en Banco Chinchorro fue abundante.

Existe coincidencia en el número de familias y géneros encontrados en otras áreas del Caribe. En Guadeloupe, Boucher \& Gourbault (1990) encontraron 10 familias y 30 géneros, $\mathrm{Al}$ menos 12 de los 20 géneros dominantes para la cuenca de Venezuela fueron comunes en Isla Mujeres y Banco Chinchorro, (Tietjen 1984), mientras que 16 especies de nematodos listados por Lewis \& Hollingsworth (1982), pertenecieron a familias que estuvieron presentes tanto en Banco Chinchorro como en Isla Mujeres. Dentro de las familias dominantes están Chromadoridae, Cyatholaimidae, Xyalidae y Comesomatidae. No obstante lo anterior, es posible que las diferencias entre las áreas geográficas, tanto para el caribe mexicano y los otros sitios mencionados estén gobernadas principalmente por las características ambientales, pero sobre todo, por el tipo de sedimento presente y el contenido de materia orgánica.

Un grupo de especies consideradas cosmopolitas también se encontraron en nuestro material. Ellas son: Desmoscolex cosmopolites, Dichromadora geophila, Enoplus communis, Spilophorella paradoxa, Spirinnia parasitifera, Terschellingia longicaudata y Trypiloides marinus, las cuales se han encontrado en casi todos los mares del mundo, tanto en áreas costeras como en la zona oceánica.

\section{Agradecimientos}

A José Juan Oliva Rivera, por su colaboración en el trabajo de campo. A Juan Pablo Carricart y a los revisores anónimos del artículo, sus comentarios y observaciones lo mejoraron sustancialmente.

\section{Literatura citada}

Andrassy I. 1973. Nematoden aus Strand and Höhlenbiotopen von Kuba. Acta Zoologica, Academic Science Hungarian 19: 233-270.

Botosaneau L. 1970. Léxpedition biospelologique a Cuba (mars-juin 1969) organizée par les Academies des Sciences de Cuba et de Roumanie: presentation sommaire des stations explorées pour le prélevement déchantillons de faune aquatique souterraine. Travail Institute Spéologie. "Emile Racovitza" 9: 81-95.

Boucher G \& N Gourbault. 1990. Sublittoral meiofauna and diversity of nematode assemblages of Guadeloupe Islands (French West Indies). Bulletin of Marine Science 47: 448-463.

Boucher G \& M Hellèouët. 1977. Nématodes des sables fins infralittoraux de la pierre Noire (Manche Occidentale). 111. Araeolaimidae et Monhysterida. Bulletin du Muséum National d'Histoire Naturelle, Serie 3 (427): 85-122.

Buchanan JB. 1984. Sediment analysis, pp. 41-65. En: Holme A \& R McIntyre (eds). Methods for the study of marine benthos. Blackwell, London.

Castillo D \& PJ Lambshead. 1992. Revision of the genus Elzalia Gerlach, 1957 (Nematoda: Xyalidae) including three new species from oil producing zones in the Gulf of Mexico, with a discussion of sibling species problem. Bulletin British Museum Natural History (Zoology) 56: 63-71.

Castillo D \& W Decramer. 1993. Cheironchus paravorax n. sp. and Cheironchus vorax Cobb, 1917 from the Campeche Sound, an oil producing zone in the Gulf of Mexico (Nemata: Selachinematidae). Bulletin de 1'Institute Royal des Sciences Naturelles de Belgique, Biologie 63: 55-64.

Chitwood BG. 1936. Some marine nematodes from North Carolina. Proceedings of Helminthology Society of Washington 3: 1-16.

Dean WE. 1974. Determination of carbonate and organic matter in calcareous sediments and sedimentary rocks by loss on ignition: comparison with other methods. Journal of the Sedimentary Petrology 448(1): 242-248.

Decramer W \& N Gourbault. 1986. Marine nematodes from Guadeloupe and other Caribbean Islands. II Draconematidae. Zoologica Scripta 15: 107-118.

Decramer W \& N Gourbault. 1987. Marine nematodes fron Guadeloupe and other Caribbean Islands. VII. The genus Epsilonema (Epsilonematidae). Bulletin de l'Institut Royal des Sciences Naturelles de Belgique 57: 57-77. 
de Jesús-Navarrete A. 1993a. Distribución, abundancia y diversidad de los nematodos (Phylum Nematoda) bénticos de la Sonda de Campeche, México. Enero 1987. Revista de Biología Tropical 41: 57-63.

de Jesús-Navarrete A. 1993b. Nematodos (Nematoda) de la laguna de Buena Vista, Quintana Roo, México. Revista de Biología Tropical 41: 649-652.

de Jesús-Navarrete A \& J Herrera-Gómez. 1999. Nematofauna asociada a la zona urbana de la bahía de Chetumal, Quintana Roo, México. Revista de Biología Tropical 47(4): 867-875.

de Jesús-Navarrete A \& J Herrera-Gómez. 2002. Vertical Distribution and feeding type of nematodes from Chetumal Bay, Quintana Roo, Mexico. Estuaries 25(6): 1131-1137.

Gerlach SA. 1963. Freilenende Meeresnematoden von den Malediven II. Kieler Meeresforsch. 19: 67-103.

Gerlach SA. 1953. Die Nematodenbesiedlung des Sandstrandes und des Küstengrundwassers an der italienischen Küste I. Systematischer Teil, Archo Zoolgie Italian 37: 517-640.

Gourbault N \& W Decramer. 1986. Nematodes marins de Guadeloupe. III. Epsilonematidae des genres nouveaux Metaglochinema n.g. (Glochinematidae) et Keratonema n. g. (Keratonematidae) n. sufam.). Bulletin Institute Museum Naturelle Paris 4, Serie 8A: 171-183.

Gourbault N \& W Decramer. 1987. Nematodes marins de Guadeloupe. VI. Les genres Bathyepsilonema et Leptepsilonema (Epsilonematidae). Bulletin Institute Museum Naturelle Paris 4, Serie 9A: 605-631.

Gourbault N \& W Decramer. 1988. Nematodes marins de Guadeloupe VIII. Le genre Perepsilonema (Epsilonematidae). Bulletin Institute Museum Naturelle Paris 4, Serie 10A: 535-551.

Gourbault N \& M Vincx. 1990. Two new species of brood protecting Desmodoridae (Nematoda) from Guadeloupe. Nematologica 36: 131-143.

Hopper BE. 1963. Marine Nematodes from the coast line of the Gulf of México. Canadian Journal of Zoology 41: 841-863.

Hopper BE. 1967. Free-living marine nematodes from Biscayne Bay, Florida, II. Oncholaimidae: description of five new species and one new genus (Meyersia). Marine Biology 1: 145-151.

Jensen P. 1986. Nematode fauna in the Sulphide-rich brine Seep and adjacent bottoms of the East Flower Garden,
N.W. Gulf of Mexico, IV. Ecological aspects. Marine Biology 92: 489-503.

Keppner EJ. 1992. Eleven new species of free-living nematodes of the genus Halalaimus de Man, 1888 (Nematoda: Enoplida) from Florida with keys to the species. Gulf Research Report 8(4): 333-362.

Keppner EJ. 1988. Six new species of free-living marine nematodes (Nematoda: Araeolaimida: Enoplida) from two estuaries in Northwest Florida, USA. Transactions of American Microscopy Society 107(1): 79-95.

Lewis JB \& CE Hollingsworth. 1982. Leaf epifauna of the seagrass Thalassia testudinum. Marine Biology 71: 4149.

Platt HM \& RM Warwick. 1983. A synopsis of free-living marine nematodes, Part I. British Enoplids, 307 pp. Cambridge University Press, London.

Renaud-Mornant J \& N Gourbault. 1981. Premieres prospections faunistiques en Martinique. I. Les biotopes et leurs peuplements. Bulletin du Muséum National d'Histoire Naturelle 4, Serie 5A: 221-234.

Soetaert K \& M Vincx. 1987. Six new Richtersia species (Nematoda, Selachinematidae) from the Mediterranean Sea. Zoologica Scripta 16: 125-142.

Tarjan AC. 1980. Marine Nematodes, 135 pp. Institute of Food and Agricultural Sciences, University of Florida, Gainesville.

Tietjen JH. 1984. Distribution and species diversity of deepsea nematodes in the Venezuela Basin. Deep Sea Research 31: 61-73.

Tietjen JH. 1989. Ecology of deep sea nematodes from the Puerto Rico Trench area and Hatteras Abyssal Plain. Deep Sea Research 36: 1579-1594.

Timm RW. 1970. A revision of the nematode order Desmoscolecida Filipjev, 1929, 115 pp. University of California Press. Berkeley, Los Angeles, London.

Wagenaar Hummerlinck P. 1977. Marine localities. Studies of the fauna of Curacao and other Caribbean Islands. Foundation for Research in Suriname and Netherland, Antilles Utrech 167: 15-68.

Wieser W \& B Hopper 1967. Marine nematodes of the east coast of North America. I. Florida. Bulletin of the Museum of Comparative Zoology, Harvard 135(5): 239334. 